\title{
Reactive Oxygen Species Generated by CD45- Cells Distinct from Leukocyte Population in Platelet Concentrates Is Correlated with the Expression and Release of Platelet Activation Markers during Storage
}

\author{
Mehran Ghasemzadeh ${ }^{a, b} \quad$ Ehteramolsadat Hosseini ${ }^{a} \quad$ Amin Shahbaz Ghasabeh ${ }^{a}$ \\ Kamran Mousavi Hosseini ${ }^{a}$ \\ ${ }^{a}$ Blood Transfusion Research Center, High Institute for Research and Education in Transfusion Medicine, Tehran, Iran; \\ ${ }^{b}$ Australian Centre for Blood Diseases, Monash University, Melbourne, VIC, Australia
}

\section{Keywords \\ CD45 antigen - Leukocyte - Platelet activation . Platelet concentrates · Reactive oxygen species}

\section{Summary}

Background: Platelet stimulation with agonists is accompanied by the generation of reactive oxygen species (ROS) which promotes further platelet activation and aggregation. Considering different cell populations in platelet concentrates (PCs), this study investigates the correlation of ROS generation with the expression and release of platelet activation markers during storage. Methods: Samples obtained from 6 PCs were subjected to flow cytometry and ELISA to evaluate the expression and shedding of platelet P-selectin or CD40L during storage. Intracellular ROS were detected in either CD45or CD45+ population by flow cytometry using dihydrorhodamine 123, while ROS production was analyzed in both P-selectin+ or P-selectin- and CD40L+ or CD40Lpopulations. To further evaluate the correlation between ROS generation and release function, TRAP-stimulated platelets were also subjected to flow cytometry analysis. Results: ROS detected in the CD45- population (leukocyte-free platelets) was significantly increased by fMLP and PMA. P-selectin- or CD40L- platelet did not show significant amount of ROS. Total ROS generation was significantly increased during platelet storage (day 0 vs. day $5 ; p=0.0002$ ) while this increasing pattern was directly correlated with the expression of P-selectin $(r=$ $0.72 ; p=0.0001)$ and CD40L $(r=0.69 ; p=0.0001)$. ROS generations were significantly correlated with ectodomain shedding of these pro-inflammatory molecules. Conclusion: Our data confirmed increasing levels of intracellular ROS generation in both platelets (CD45-) and platelet-leukocyte aggregates (CD45+) during PC storage. The amount of detected ROS is directly correlated with platelet activation and release in each population while platelet-leukocyte aggregates generate higher levels of ROS than single platelets.

(c) 2018 S. Karger GmbH, Freiburg

\section{Introduction}

Although the in vivo shelf life of platelets in circulation is about 8-10 days, platelet components cannot be stored more than 5 days due to the risk of bacterial contamination and some deleterious changes that cause progressive structural and functional damage in the cells, the so-called platelet storage lesion (PSL) [1-3].

In platelet components, PSL is commonly manifested by reduced $\mathrm{pH}$, due to glycolysis and increased levels of lactate, platelet cytoskeleton rearrangement leading to platelet shape change, and abnormal aggregation function with increased expression of active $\alpha_{\mathrm{IIb}} \beta_{3}$ as well as secretory granule release that induce pro-inflammatory phenotype of platelets expressing P-selectin and CD40L. Finally, increased levels of intracellular calcium and externalization of phosphatidylserine induce pro-coagulant platelets that progres-

\section{KARGER}

(C) 2018 S. Karger GmbH, Freiburg 
sively shift to an apoptotic phenotype characterized by caspase activity and loss of mitochondrial membrane potential in long-stored platelets [4-6]. Unwilling activation of platelets during storage causes granule release associated with the increased expression and shedding of P-selectin and CD40L as important pro-inflammatory molecules and specific markers of platelet activation [7].

In stored platelets, irreversible shift of the pro-aggregatory phenotype to the pro-inflammatory state may dramatically affect platelet function and fate in circulation via the interaction and/or conjugation of P-selectin expressing platelets with leukocytes holding P-selectin glycoprotein ligand-1. Notably, in addition to the induction of unwilling premature inflammatory responses, such platelets may be rapidly removed by scavenging pathways including reticuloendothelial system [8-10]. These problems reduce the efficacy of platelet transfusion therapy. Stored platelets also express significant levels of CD40L which was shown to trigger pro-inflammatory pathways via the interaction with different types of leukocytes $[11,12]$.

Several lines of evidence also showed that platelet activation is associated with increased levels of reactive oxygen species (ROS) [13-15]. Therefore, like other activation markers, ROS generation may also reflect the state of activity of platelets. However, as unstable components that rapidly react with other proteins including signaling molecules, ROS interfere in platelet function through the modification of the signaling pathway. In physiologic concentrations, ROS usually act as intracellular signals resulting in platelet activation [16]. Among several enzymatic pathways which elicit ROS formation, NADPH oxidase (NOX2) is mainly involved in the generation of $\mathrm{O}_{2}^{-}$, an unstable product which is rapidly converted to $\mathrm{H}_{2} \mathrm{O}_{2}$ by the superoxide-dismutase (SOD). Studies showed that upon platelet stimulation, NOX-dependent generation of different components of ROS, including $\mathrm{O}_{2}^{-}$and $\mathrm{H}_{2} \mathrm{O}_{2}$, results in propagation of platelet activation and aggregation [17-19].

Platelet stimulation with different agonists such as thrombin increase ROS generation [13]. Collagen-induced aggregation also showed to enhance ROS generation via eliciting the considerable amount of $\mathrm{H}_{2} \mathrm{O}_{2}$ which mobilizes intracellular calcium. This ROSdependent calcium influx induces further platelet activation via the activation of phospholipase A2 (PLA2), membrane release of arachidonic acid, and thromboxane A2 generation as well as phospholipase $\mathrm{C}$ up-regulation [15]. Consistently, it has been shown that NOX-dependent platelet $\mathrm{O}_{2}{ }^{-}$generation promotes platelet aggregation and thrombosis by increasing platelet recruitment to the site of injury [20,21].

Several lines of evidence showed an intimate crosstalk between ROS and pro-inflammatory mediators. Signals induced by ROS augment the production and release of pro-inflammatory cytokines which perpetuate several inflammatory responses [22-24]. On the other hand, the pro-inflammatory status also enhances ROS generation in different conditions. The most relevant example is the involvement of CD40L in ROS generation in neutrophils, shifting these cells to their active inflammatory state $[25,26]$.

Given the important role of ROS in propagation of platelet activation, the aim of the present study was to elucidate whether plate- let storage can significantly result in an increasing amount of ROS production and whether the generated ROS is correlated with the increased levels of platelet pro-inflammatory molecules.

\section{Material and Methods}

Reagents

Mouse IgG1 к Isotype controls (phycoerythrin(PE)-, fluorescein isothiocyanante(FITC)- and peridinin chlorophyll protein complex(PerCP)-conjugated) were from Miltenyi Biotec (Bergisch Gladbach, Germany). Monoclonal antibodies against human CD154 (PE- or FITC-conjugated), CD45 (PE-conjugated), CD42b (FITC- or PE-conjugated) and CD62P (PerCP-conjugated) were from BD Pharmingen (San Diego, CA, USA). Dihydrorhodamine (DHR) 123 and other reagents and chemicals were from Sigma Aldrich (St. Louis, MS, USA).

\section{Sample Preparation}

The present study was conducted on the samples of 6 blood donors. The study was approved by the local ethical committee, and informed consent was obtained from blood candidates by the Iranian Blood Transfusion Organization (IBTO). Six platelet-rich plasma platelet concentrate (PRP-PC) bags obtained from the volunteer donors were randomly selected. All platelet concentrates (PCs) passed release process under IBTO screening regulations. Each bag included 50-60 ml PCs with more than $1 \times 10^{9}$ platelets $/ \mathrm{ml}$. On day 0 of storage, 5 $\mathrm{ml}$ of product was taken under sterile conditions from the cord for the baseline study and after sealing back using a connecting device instrument (TSCD-II; Terumo, Tokyo, Japan), original bags of PCs were connected to 3 small satellite bags with the same origin. PRP-PCs were then equally portioned to the small bags using a digital balance (Sartorius, Göttingen, Germany). The three small bags originated from each primary PCs were labeled as day 1, day 3, and day 5 . Prior to sample preparation, all bags were kept in a shaker incubator at $20-24{ }^{\circ} \mathrm{C}$. PRP-PCs were transferred to falcon tubes, and by adding a designated amount of Tyrode's buffer ( $10 \mathrm{mmol} / \mathrm{l}$ Hepes, $12 \mathrm{mmol} / \mathrm{NaHCO}_{3}, \mathrm{pH}$ 7.4, $137 \mathrm{mmol} / \mathrm{l} \mathrm{NaCl}, 2.7 \mathrm{mmol} / \mathrm{l} \mathrm{KCl}, 5 \mathrm{mmol} / \mathrm{l}$ glucose, $1 \mathrm{mmol} / \mathrm{C} \mathrm{CaCl}_{2}$ ), platelet counts were adjusted to $5 \times 10^{8} / \mathrm{ml}$. Washed PCs were isolated as described previously [27].

For flow cytometry analysis, platelet counts were adjusted to $10^{7} / \mathrm{ml}$. Platelet-poor plasma (PPP) obtained from PRP with a platelet count of $5 \times 10^{8} / \mathrm{ml}$ was subjected to two-step ultracentrifugation $\left(3 \times 10^{4} \mathrm{~g}\right.$ for $30 \mathrm{~min}$ each time) and microparticle-free supernatant was separated and kept at $-20{ }^{\circ} \mathrm{C}$ to be analyzed by ELISA assay for the evaluation of shed proteins. To prepare resting platelets for control study, blood sample was drawn from healthy consenting individuals who had not taken any anti-platelet or anti-inflammatory medication in the 2 weeks prior to venesection, and platelets were isolated as already described $[27,28]$.

\section{Analysis of Platelet Oxidative Burst (ROS Generation)}

DHR 123 was already reported to be used for evaluation of ROS production in platelets [29-31]. DHR 123 is a cell-permeable mitochondrial-avid component that can undergo oxidation to the fluorophore rhodamine 123 [32]. The oxidized rhodamine has a tendency to remain within the cell after tautomerization of its equivalent amino groups. DHR 123 provides the advantage of being oxidized by both $\mathrm{H}_{2} \mathrm{O}_{2}$ and $\mathrm{O}_{2}^{-}$while emitting a strong fluorescence signal. This makes it a very sensitive indicator for detection of ROS generation [33]. To analyze ROS production, platelets $\left(10^{7} / \mathrm{ml}\right)$ loaded with $1 \mu \mathrm{mol} / \mathrm{l} \mathrm{DHR} 123$ for $30 \mathrm{~min}$ at $37^{\circ} \mathrm{C}$ were incubated with PE-conjugated anti-CD45 (as a leukocyte marker) in the presence and absence of either $2 \mu \mathrm{mol} / \mathrm{l} \mathrm{fMLP}$ (N-formylmethionyl-leucyl-phenylalanine) or $200 \mathrm{nmol} / \mathrm{l}$ PMA (phorbol 12-myristate 13-acetate). Cells were then subjected to flow cytometry (CyFlow ${ }^{\circledR}$ Space, Sysmex Partec GmbH, Görlitz, Germany) where a total of 20,000 platelet events were acquired. The flow cytometer settings were optimized for the acquisition of 

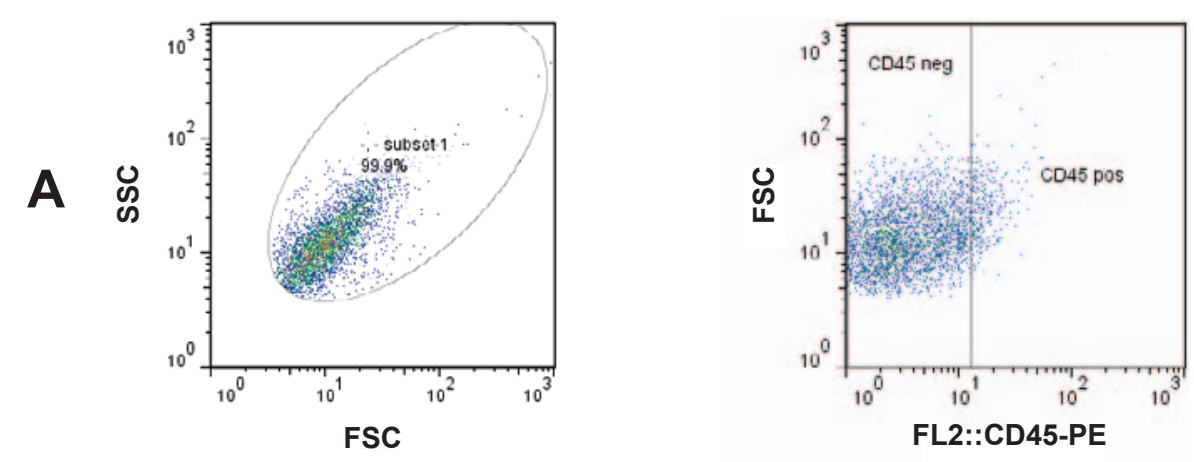

Fig. 1. DHR 123 expression in leukocyte and platelet population in PRP-derived platelets. Platelets from day 1 were loaded with $1 \mu \mathrm{mol} / 1$ DHR 123 for $30 \mathrm{~min}$ at $37^{\circ} \mathrm{C}$, and then incubated with PEconjugated anti-CD45. DHR 123 expression in either CD45+ (leukocyte or platelet-leukocyte aggregate) or CD45- (platelets alone) populations were detected (A, B). A representative dotted plot illustrates DHR 123+ events in CD45- population compare to that of control $(\mathbf{C})$. DHR $=$ Dihydrorhodamine.
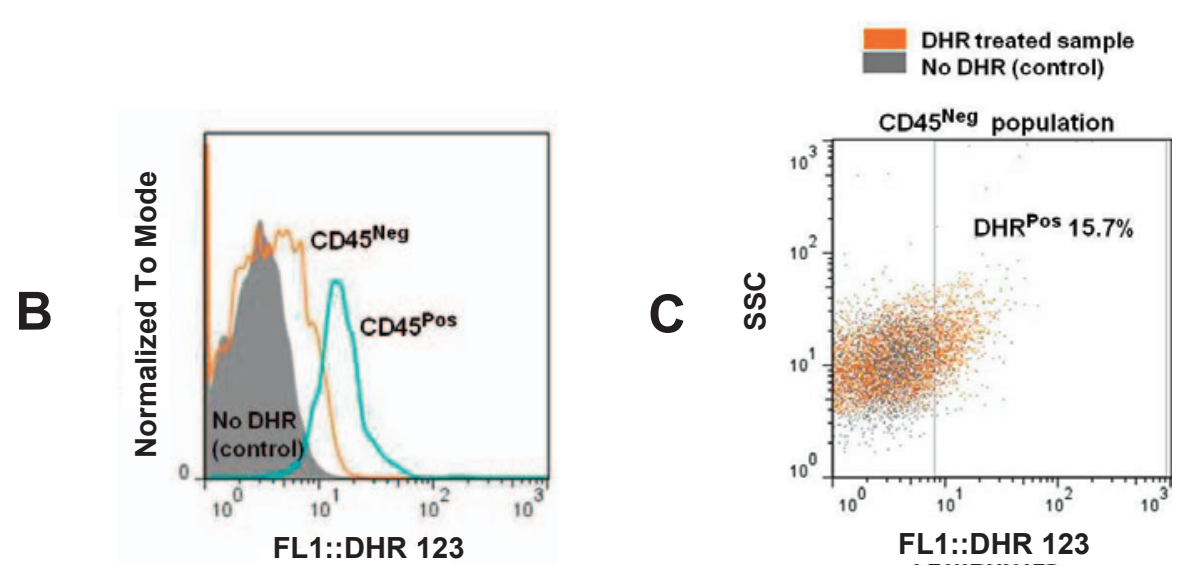

platelets by logarithmic signal amplification in all 3 detectors (forward and side scatter channels and fluorescence channel FL1). For analysis, the gate was set around the intact platelet population (R1) as defined by forward and side scatter characteristics and confirmed by the presence of platelets expressing GPIba (in a parallel experiment) with the least expression of CD45 (using anti-CD45 in dual staining with DHR 123). To verify platelet-originated ROS production for each run, dual staining of DHR 123 with CD45 was performed so that the $\mathrm{CD} 45+$ population of platelet-leukocyte aggregate (R2) could be excluded from our gating strategy for platelet-originated ROS. The percentage of platelets expressing DHR 123 fluorescence in comparison with the background (negative control) was recorded. Data were analyzed with FLOWJO software (Tree Star Inc., Ashland, OR, USA). Using platelet dual staining with either DHR 123 and CD40L (PE-conjugated) or DHR 123 and P-selectin (PerCP-conjugated), the percentage of DHR 123+ cells in either P-selectin- or CD40L+ and P-selectin or CD40L- populations were also evaluated.

\section{Flow Cytometry to Determine the Levels of Surface Molecules Expression}

Platelets were stained with anti-P-selectin, anti-CD40L and anti-CD42b (GPIba) for $30 \mathrm{~min}$. Isotype-matched control antibodies were used to determine background staining created by non-specific binding. Cells were then fixed in $1 \%$ paraformaldehyde in phosphate-buffered saline for $1 \mathrm{~h}$ and subjected to flow cytometry (CyFlow ${ }^{\circledR}$ Space) where a total of 20,000 platelet events were acquired. The flow cytometer settings were optimized for the acquisition of platelets by logarithmic signal amplification in all four detectors (forward and side scatter channels and fluorescence channels FL1 and FL2). For analysis, the gate was set around the intact platelet population as defined by forward and side scatter characteristics and confirmed by the presence of platelets expressing GPIba. The percentage of positive platelets expressing either P-selectin or CD40L above the background (negative control) was recorded. Data were analyzed with FLOWJO software.
Enzyme-Linked Immunosorbent Assay

Microparticle-free samples obtained from PPP were subjected to ELISA assay to determine the exact concentration of soluble P-selectin and CD40L using ab100631-CD62P and ab9999-CD40L Human ELISA Kits (Abcam, Cambridge, UK), respectively, according to the manufacturer's instructions. The absorbance at $450 \mathrm{~nm}$ was read with an ELISA reader (Multiskan EX, Labsystem, Helsinki, Finland).

\section{Platelet Counting and pH Measurement}

Platelet count and mean platelet volume (MPV) were measured using a hematology full blood analyzer (XE-2100, Sysmex, Milton Keynes, UK) on days 0, 1,3 , and 5 . Measurement of $\mathrm{pH}$ was performed at $22^{\circ} \mathrm{C}$ on a pH meter $(826 \mathrm{pH}$ mobile / 827 pH lab, Metrohm AG, Herisau, Switzerland).

\section{Bacterial Cultures}

Bacterial cultures were performed on day 0 and 5 for each bag of platelets, including both aerobic and anaerobic cultures.

\section{Statistical Analysis}

For comparison of the expression and shedding of platelet surface molecules in different days, data were analyzed by KruskalWallis test with Dunn's multiple comparison test. For correlations, linear regression analysis was applied. $\mathrm{P}$ values of less than 0.05 were considered to be significant. Calculations were done using GraphPad Prism software (GraphPad Prism Software, Inc., San Diego, CA, USA). 
A
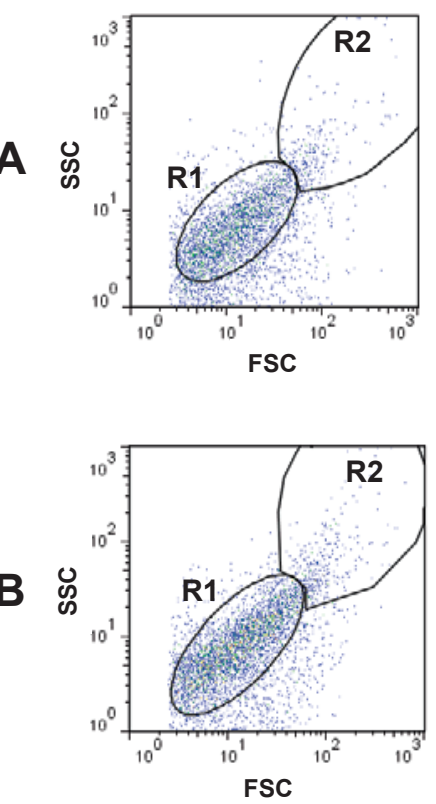

ROS in R1 population

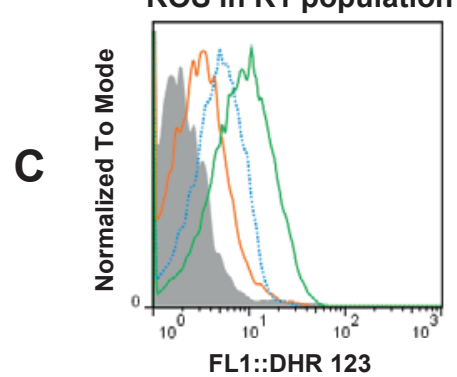

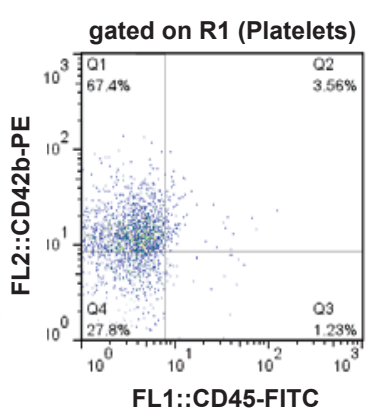

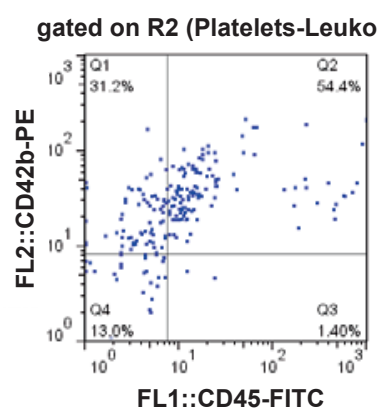

Platelet-Leukocyte (R2)

Platelet (R1)

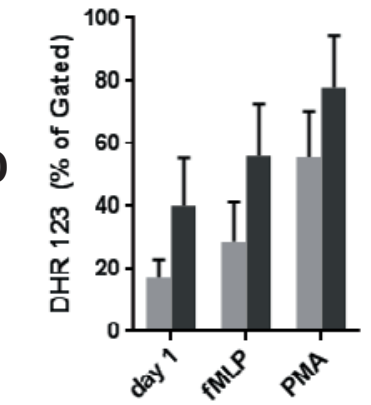

Platelet(R1)

Hlatelet-leukocyte(R2)

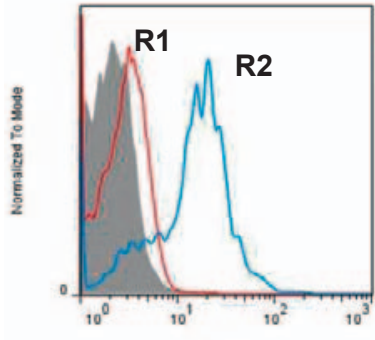

FL2::CD45-PE ROS in $\mathbf{R} 2$ population

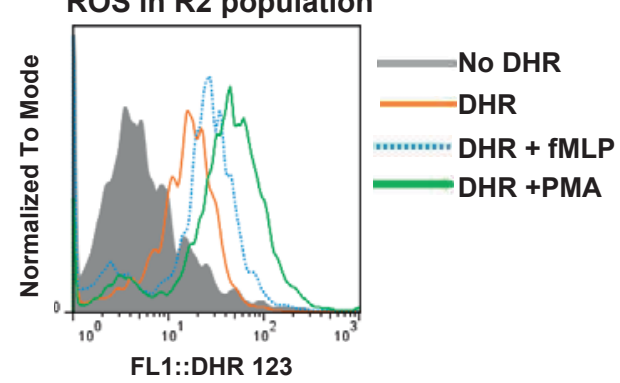

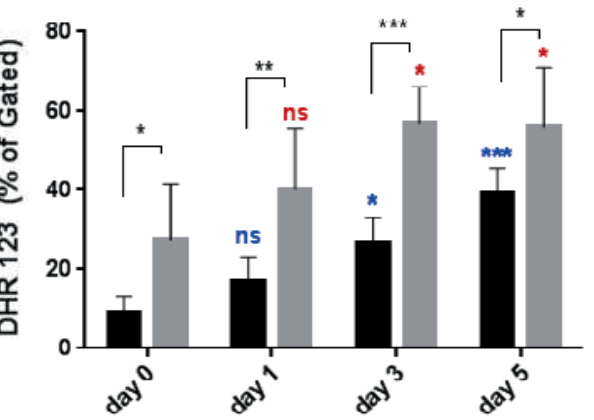

Fig. 2. ROS generation in single platelets versus platelet-leukocyte population in PCs: Platelets on day 1were loaded with $1 \mu \mathrm{mol} / 1 \mathrm{DHR} 123$ for $30 \mathrm{~min}$ at $37^{\circ} \mathrm{C}$, and then incubated with PE-conjugated anti-CD45 in the presence and absence of either $2 \mu \mathrm{mol} / \mathrm{l} \mathrm{fMLP}$ or $200 \mathrm{nmol} / \mathrm{l} \mathrm{PMA}$. B CD45 demonstrated the lowest levels in R1 (single platelet area), whereas highest levels of leukocytes were found in theR2 area. C Representative histograms show DHR 123 expressions in areas (R1 or R2), ROS generation was increased in the presence of fMLP or PMA . D Increased levels of DHR 123 expression in responses to mentioned agonists in 6 PCs after one day storage. $\mathbf{E}$ Levels of ROS production in R1 versus R2 during storage of 6 units of PCs. DHR = Dihydrorhodamine; PCs = platelet concentrates; ROS = reactive oxygen species. ${ }^{* *} \mathrm{p}<0.001,{ }^{* *} \mathrm{p}<0.01,{ }^{*} \mathrm{p}<0.05, \mathrm{~ns}=$ not significant $\mathrm{p}>0.05$.

\section{Results}

\section{Platelet Count, Size, and $p H$}

Platelet counts and size were measured on days $0,1,3$, and 5 . The platelet counts decreased after 5 days, but the decrease was too small to have any major influence on the quality of the products. The MPV of platelets increased significantly on day 5 compared with day 1 . The $\mathrm{pH}$ was generally stable during storage (data not shown).

\section{ROS Production in Platelet Population Detected by DHR 123}

PCs stored for 1 day were subjected to flow cytometry analysis using dual staining protocol to detect DHR 123 expression in either CD45+ (leukocyte or platelet-leukocyte aggregate) or CD45(platelets alone) populations. As showed in figure 1, 7\% of population was CD $45+$ of which $88 \%$ expressed DHR 123 whereas in population which was CD45-, 15\% expressed DHR 123. To fur- ther investigate ROS generation in these two distinct populations, platelet-leukocyte and/or leukocyte populations (R2: 6\%) were discriminated from platelets (R1: 75\%) using dual staining of platelet concentrate with PE-conjugated anti-CD42b (as a platelet marker) and FITC-conjugated anti-CD45 (fig. 2A). In a parallel study using dual staining of PCs with DHR 123 (FL1) and PE-conjugated antiCD45 (FL2), DHR 123 expressions in the same areas (R1 or R2) were analyzed, which confirmed ROS generation in platelet population (CD45-) although at lower levels than platelet-leukocyte/ leukocyte population (CD45+) (fig. 2B). In addition, as shown in figure $2 \mathrm{C}$, ROS generation in either platelet population (CD45-) or platelet-leukocyte/leukocyte population $(\mathrm{CD} 45+)$ was also significantly increased in the presence of fMLP $(2 \mu \mathrm{mol} / \mathrm{l})$ or PMA (200 nmol/l). Figure 2D shows DHR 123 expression of either single platelets or platelet-leukocyte/leukocyte population in response to above mentioned agonists in 6 PCs after 1 day of storage. These results validated the reproducibility and efficacy of flow cytometry- 
A
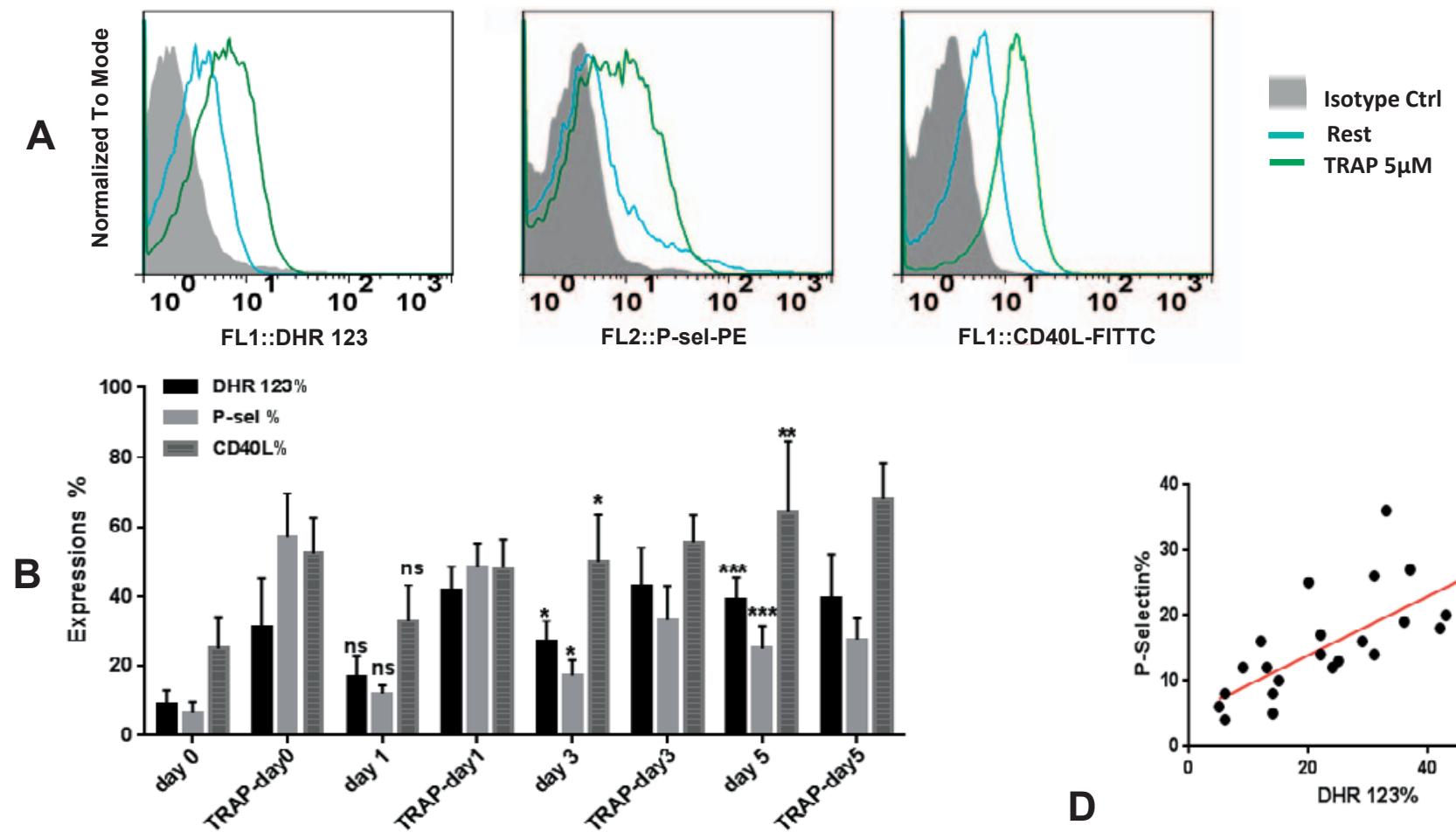

D
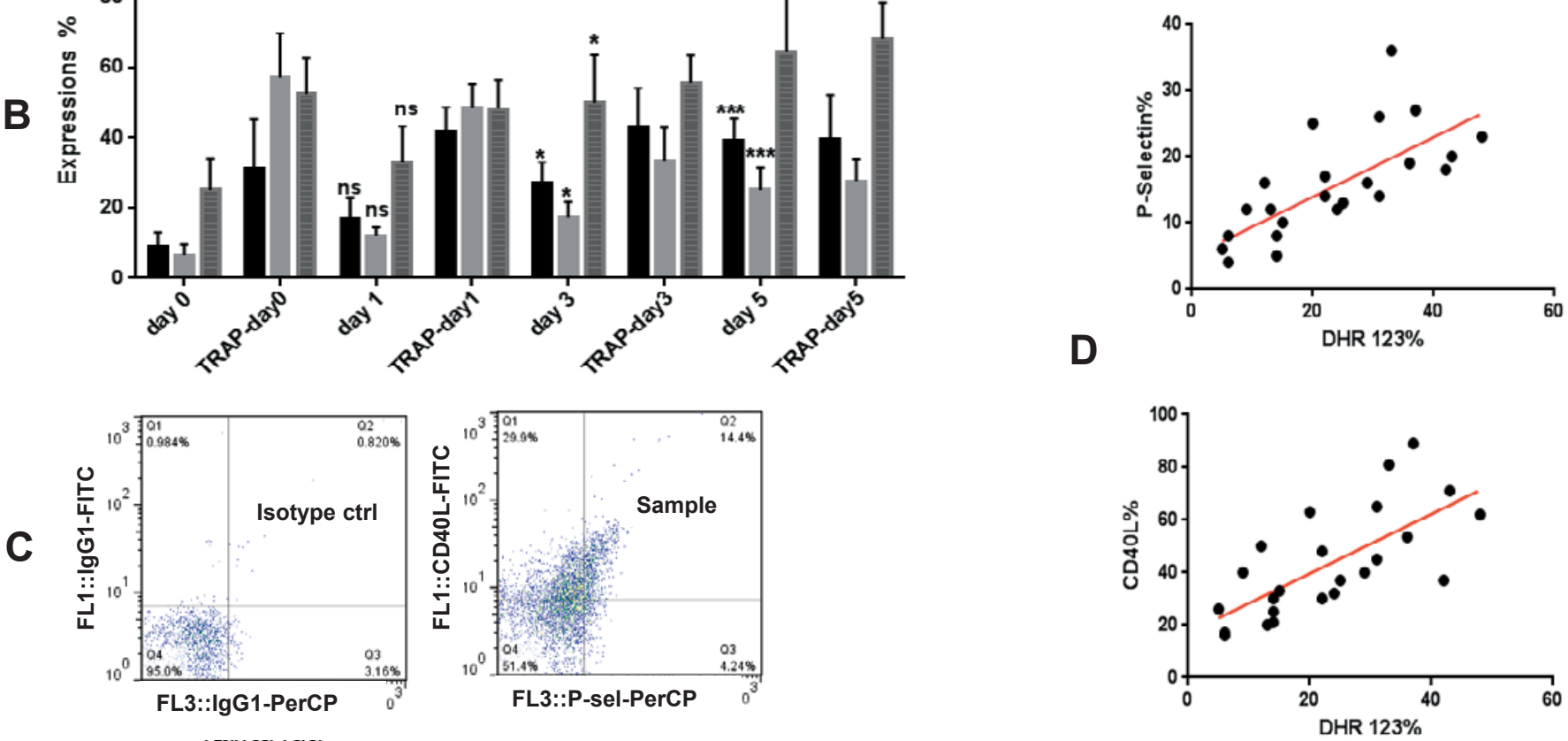

Fig. 3. ROS generation in PRP-derived platelets correlated with either P-selectin or CD40L expression during 5 days storage. Platelets were either loaded with $1 \mu \mathrm{mol} / \mathrm{l}$ DHR 123 for $30 \mathrm{~min}$ at $37^{\circ} \mathrm{C}$ or stained with both PerCP conjugated anti-P-selectin and FITC conjugated anti-CD40L. Representative histograms (A) and Graph (B) show the basal levels of expressions for DHR 123, P-selectin and CD40L in addition to those levels in response to TRAP (5 $\mu$ mol/1) on days 0, 1, 3, and 5 of storage. Representative dotted plot (C) demonstrates P-selectin+/CD40L+ population on day 3. D shows direct correlation between the expression levels of either P-selectin $(\mathrm{r}=0.72 ; \mathrm{p}=0.0001)$ or CD40L $(\mathrm{r}=0.69 ; \mathrm{p}=0.0001)$ and DHR 123 expression in stored platelets. DHR $=$ Dihydrorhodamine; ROS $=$ reactive oxygen species. ${ }^{* *} \mathrm{p}<0.001,{ }^{* *} \mathrm{p}<0.01,{ }^{*} \mathrm{p}<0.05$, ns $=$ not significant $\mathrm{p}>0.05$.

based DHR 123 expression tests for evaluating of platelet-originated ROS generation during PC storage. PCs were also examined for platelet-originated ROS generation during storage at days 0,1 , 3 , and 5 . As shown in figure $2 \mathrm{E}$, ROS generation of platelets significantly increased from day 0 to day $5(\mathrm{p}<0.001)$. A similar pattern of ROS generation was also observed in the platelet-leukocyte/leukocyte population during storage. However, the increasing levels of ROS generation from day 0 to day 5 were less significant here than those in platelets $(\mathrm{p}<0.05)$.

\section{P-selectin and CD40L Expression Correlate with Platelet ROS \\ Generation during Storage}

To investigate the association of ROS production with platelet activation and granule release, the expression of each pro-inflammatory molecules was monitored during PC storage using dual staining of platelets with PerCP-conjugated anti-P-selectin (FL3) and FITC conjugated anti-CD40L (FL1) (fig. 3C). As depicted in figure $3 \mathrm{~B}$, gradual increases of expression levels for both pro-inflammatory molecules were detected during storage, and they were associated with increasing levels of ROS generation in platelets. Platelet P-selectin responses to TRAP were significantly higher on days 1 and 3 after storage than at baseline, whereas on day $5 \mathrm{P}$-selectin expression in response to agonist was not significantly increased. A similar pattern was also observed for CD40L; however, from day 3 after storage, the amount of increased expression in response to TRAP was not significantly higher than at baseline. TRAP-activated platelets also revealed significantly higher levels of ROS generation than non-stimulated samples on all days of storage, except day 5 (fig. 3B). Further analysis showed a direct correlation between P-selectin $(r=0.72 ; \mathrm{p}=0.0001)$ and DHR 123 expression in platelets during storage. CD40L expression was also significantly correlated with platelet ROS generation in stored 

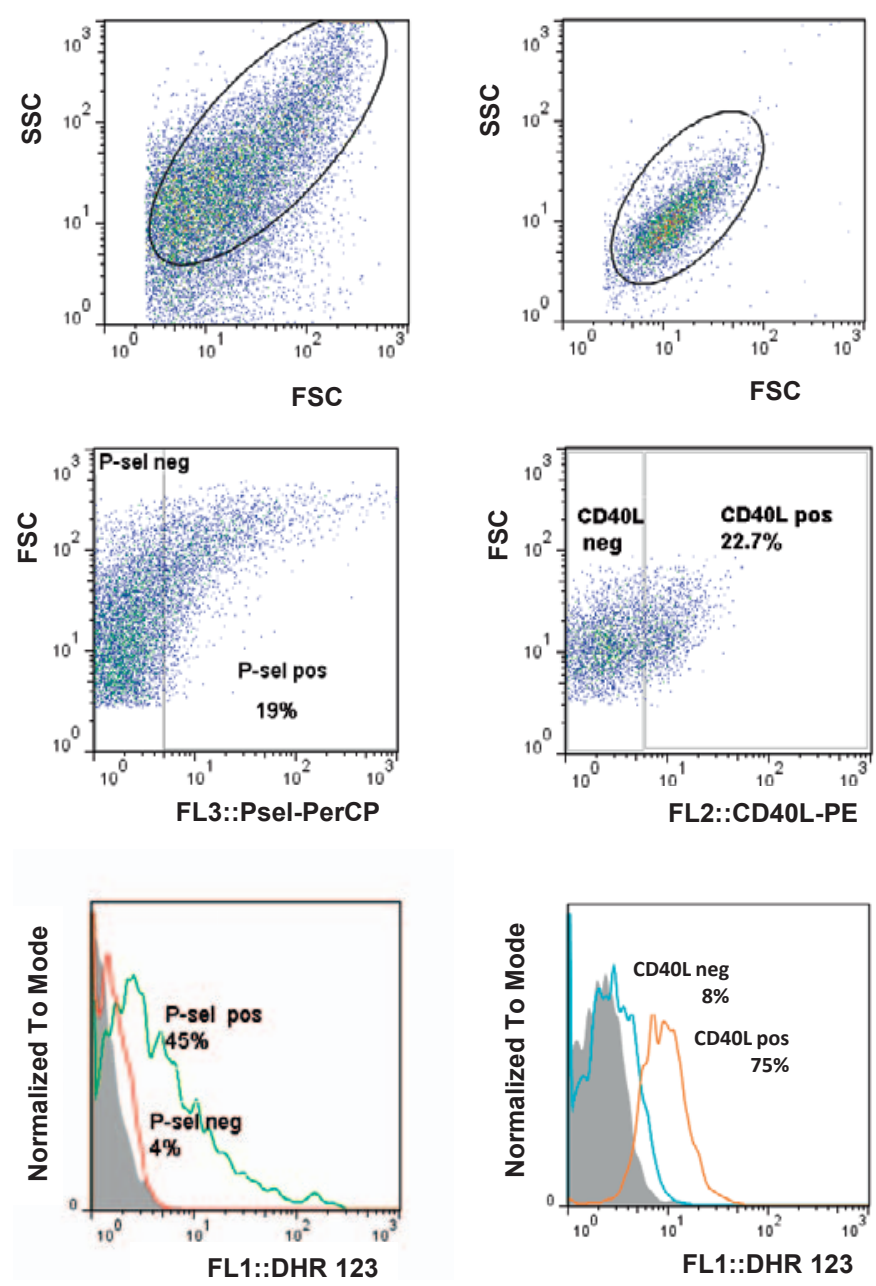

Fig. 4. ROS does not significantly express in either P-selectin- or CD40L-cell populations in stored PCs. Platelets stored for 3 days were loaded with $1 \mu \mathrm{mol} / \mathrm{l}$ DHR 123 for $30 \mathrm{~min}$ at $37^{\circ} \mathrm{C}$ and then incubated with either PerCP-conjugated anti-P-selectin or PE-conjugated anti-CD40L. The cell population first gated based on these pro-inflammatory molecules expressions. Subsequently, cells which were either positive or negative for each molecule expression were separately analyzed for DHR 123 expression. The lowest levels of ROS generation in the cells which were negative for either P-selectin or CD40L. DHR = Dihydrorhodamine; $\mathrm{PCs}=$ platelet concentrates; $\mathrm{ROS}=$ reactive oxygen species .

platelets ( $r=0.69 ; \mathrm{p}=0.0001$ ) (fig. $3 \mathrm{D})$. Furthermore, as illustrated in figure 4 , for PCs stored for 3 days which are expected to have a moderate levels of P-selectin expression ( 20\%), flow cytometry assays did not show any significant DHR 123 expression in the Pselectin or CD40L- population using platelet dual staining with either fluorochrome-conjugated anti-P-selectin or anti-CD40L and DHR 123. Conversely, platelet populations expressing each of these molecules exhibited the high levels of DHR 123, an observation that confirmed the correlation of platelet activation and release with ROS generation during storage of PCs. We also demonstrated higher levels of P-selectin expression in CD45+ (leukocyte or platelet-leukocyte aggregate) than in CD45- (platelets alone) populations (fig. 5A). In a parallel study, it has been shown that both Pselectin and DHR 123 expressions were much higher in R2 population than R1 (fig. 5B).
P-selectin and CD40L Ectodomain Shedding (Release) Correlate with Platelet ROS Generation during Storage

Like the expression results, the levels of released P-selectin and CD40L also increased during 5 days storage, while this was associated with increasing levels of DHR 123 expression in platelets (fig. 6A). As shown in figure 6B, both $\mathrm{P}$-selectin and CD40L release were directly correlated with ROS generation in platelets (soluble P-selectin: $\mathrm{r}=0.78 ; \mathrm{p}<0.0001$ and soluble CD40L: $\mathrm{r}=0.75 ; \mathrm{p}<$ $0.0001)$.

\section{Discussion}

In the present study, we investigated the relationship between platelet ROS generation and platelet pro-inflammatory states during storage of PCs for 5 days. Our results showed increasing levels of intra-platelet ROS generation during storage, which was significantly correlated with platelet expression or release of pro-inflammatory molecules such as P-selectin and CD40L. Our data also demonstrated that $\mathrm{P}$-selectin- or CD40L-expressing platelets generated ROS, whereas platelets not expressing these pro-inflammatory molecules did not produce ROS. Several studies suggested P-selectin expression and release as common markers of platelet activation during storage. As another important pro-inflammatory molecule, CD40L expression and release have been shown to be correlated with those of P-selectin during storage [7, 34]. Thus, CD40L can also be considered as an additional marker of platelet activation. On the other hand, platelet ROS generation can regulate platelet activation $[20,21]$, as surface expression of platelet activation markers, including P-selectin, CD40L or activated integrin $\alpha_{\mathrm{IIb}} \beta_{3}$, was shown to be attenuated by pharmacologic antioxidants [35]. In addition, platelet dysfunction reported in patients with genetically determined impaired $\mathrm{O}_{2}{ }^{-}$formation can provide further supporting evidence for the importance of ROS generation in eliciting platelet activation $[18,36]$. However, whether platelet oxidative changes first induce platelet activation or whether platelet stimulation with activating agonists triggers ROS generation is still not defined unambiguously [37]. Platelet storage is also associated with ROS generation. Using rat platelets suspended in Tyrode's buffer, Manasa and Vani [37] demonstrated a significant increase of superoxides after 6 days of storage. They also showed significant increment of SOD during this period while catalase activity significantly decreased after 8 days of storage. They suggested that platelets may endure oxidative stress during the 6 days of storage; however, it should be noted that this result could not be extended to the blood-banked platelets which were stored under different conditions. The authors were also able to show that platelet aggregation increased in proportion to the free radical generation during storage, which indicated that ROS generation is correlated with platelet aggregation. In another study [38] performed on PRP obtained from healthy volunteers, platelet storage time was shown to be associated with elevated generation of mitochondrial-based ROS as well as a progressive increment in apoptosis detected by platelet mitochondrial respiration and functional decline. This study indicated a significant increase in 

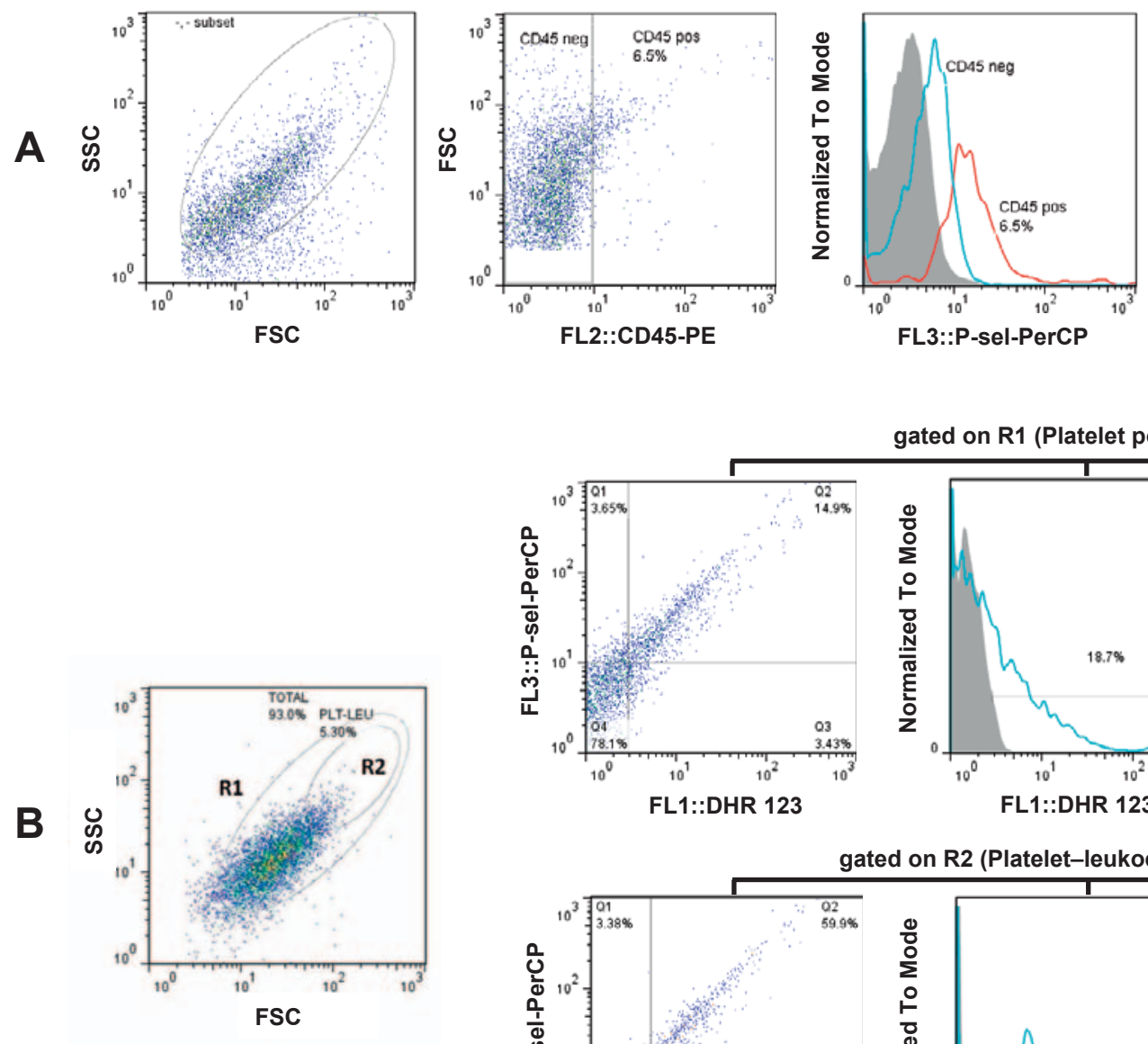

gated on R1 (Platelet population)
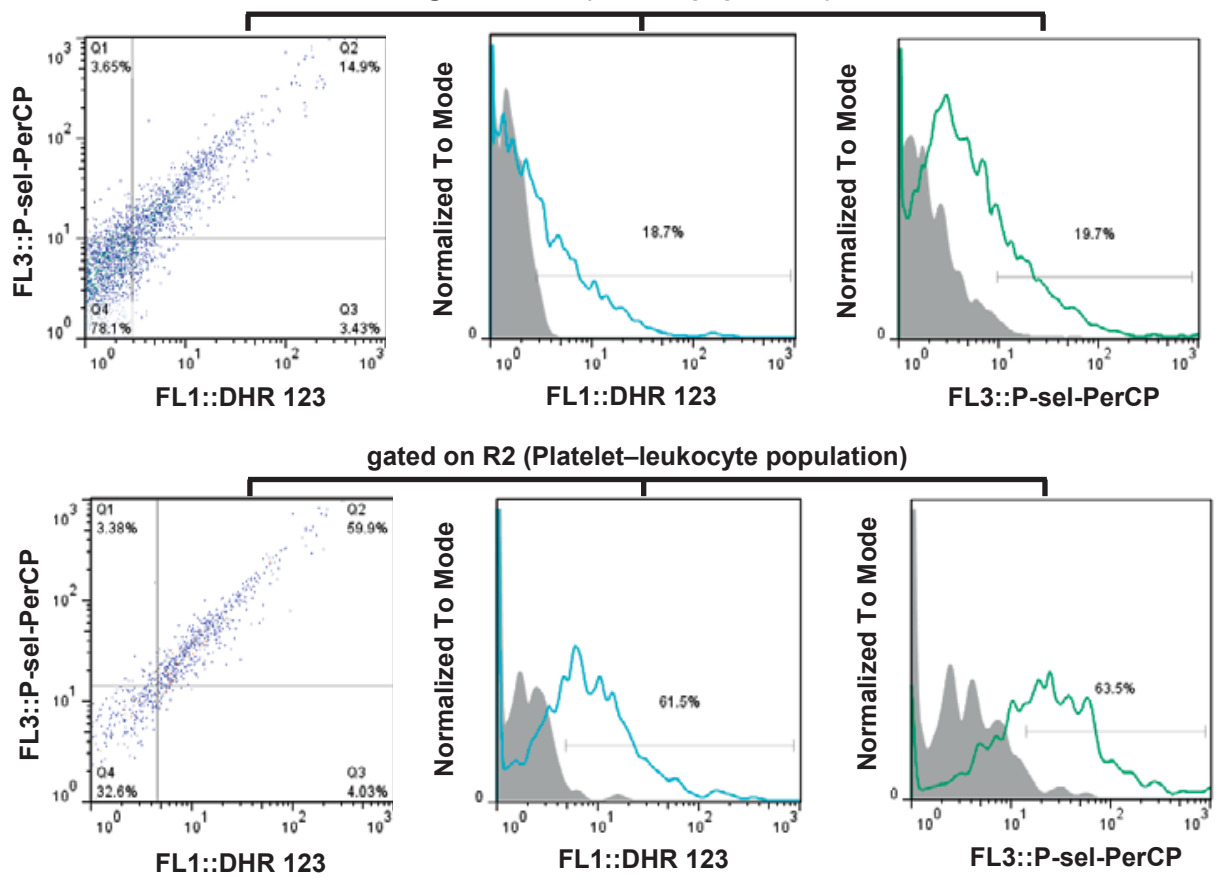

Fig. 5. Platelet-leukocyte interaction increases platelet activation leading to higher levels of ROS generation in platelet-leukocyte aggregates. A Platelets on day 3 after storage were stained with both PerCP-conjugated anti-P-selectin and PE-conjugated anti-CD45 to detect P-selectin expression in platelets and/or plateletleukocyte aggregates. B Platelets stored for 3 days (at $10^{7} / \mathrm{ml}$ in Tyrode's buffer) were loaded with $1 \mu \mathrm{mol} / \mathrm{l}$ DHR 123 for 30 min at $37^{\circ} \mathrm{C}$ and then stained with PerCP-conjugated anti-P-selectin. Histograms show DHR 123 and P-selectin expressions in areas (R1 or R2), which confirmed much lower ROS generation and platelet activation in platelet population than platelet-leukocyte/leukocyte population. $\mathrm{DHR}=$ Dihydrorhodamine; $\mathrm{ROS}=$ reactive oxygen species .

platelet mitochondrial production of ROS, 3 and 5 days after storage when compared to day 0 . In addition, Skripchenko et al. [39] also monitored intracellular ROS generation in stored PCs by flow cytometry using a modified technique of PRP isolation from whole blood. They found significant increases in platelet ROS generation 6 days after storage which was directly correlated with higher levels of carbon dioxide and lower oxygen levels. They suggested that prolonged periods of elevated carbon dioxide, potentially coupled with other platelet activation factors, are associated with platelet mitochondrial dysfunction and poor $\mathrm{pH}$ condition during storage. Both of these observations suggest that the increasing generation of intracellular ROS plays a role in platelet apoptosis and pro-coagulant functions. Whether ROS generation also plays a role in the enhancement of platelet pro-inflammatory state and granule release or whether platelet pro-inflammatory state during storage induces ROS generation was subject of our study. We therefore studied the correlation of platelet ROS generation with the expression and release of platelet pro-inflammatory molecules such as P-selectin and CD40L. Similar to other studies, we showed an increasing pattern of intra-platelet ROS during storage. As already shown by Skripchenko et al. [39], we demonstrated a significant increase in ROS production from day 3 after storage. This suggests that, contrary to the study by Manasa and Vani [37], the platelet antioxidant system prevent oxidative stress progression endures for 3 days in banked PCs under conditions of continuous agitation. It is important to know that without pre-storage leukoreduction PCs inevitably include a residual amount of leukocytes which, although their concentration is very low, may contain much higher levels of ROS than platelets, especially when they form aggregate with platelets [40]. Several lines of evidence showed that platelets expressing pro-inflammatory molecules effectively recruit different subtypes of leukocytes which can then be activated through a direct crosstalk with 
Fig. 6. ROS generation in PRP-derived platelets correlated with either P-selectin or CD40L shedding during 5 days of storage. To detect the levels of ROS generation, platelets on days $0,1,3$, and 5 of storage were loaded with $1 \mu \mathrm{mol} / 1 \mathrm{DHR} 123$ for $30 \mathrm{~min}$ at $37^{\circ} \mathrm{C}$ and subjected to flow cytometry analysis. In addition, Microparticle-free samples obtained from same PRP-PCs were also subjected to ELISA assay to determine the exact concentration of soluble P-selectin and CD40L. A Levels of shed P-selectin and CD40L during storage. $\mathbf{B}$ Direct correlation between the shedding levels of either P-selectin ( $\mathrm{r}=0.78$ $\mathrm{p}<0.0001)$ or CD40L $(\mathrm{r}=0.75 ; \mathrm{p}<0.0001)$ and ROS generation (DHR 123 expression) in stored PCs. DHR = Dihydrorhodamine; MP = microparticles; PCs = platelet concentrates; ROS $=$ reactive oxygen species. ${ }^{* *} \mathrm{p}<0.001,{ }^{* *} \mathrm{p}<0.01$, ns: not significant $\mathrm{p}>0.05$.

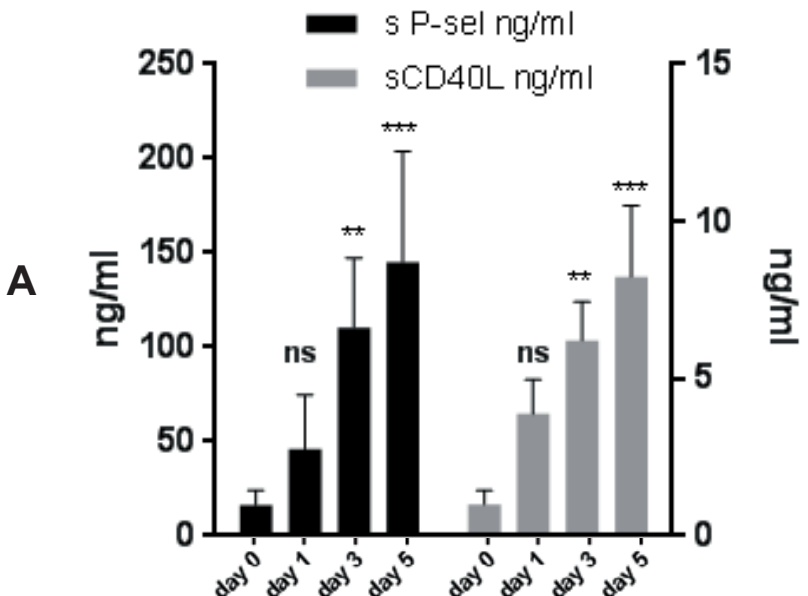

B
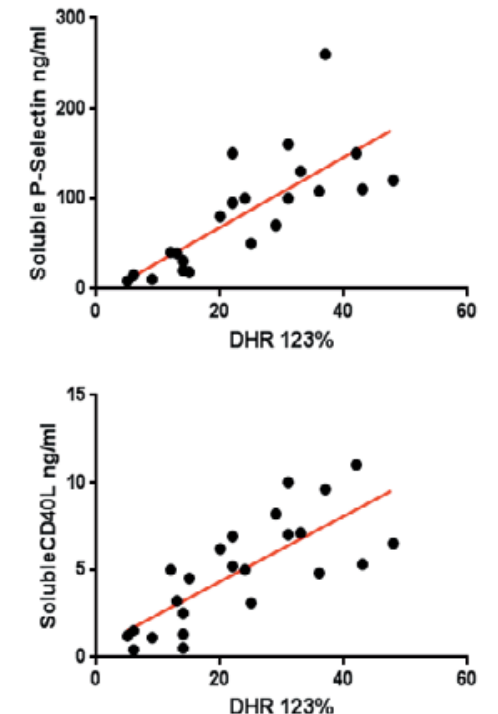

platelets while generating a considerable amount of ROS [41, 42]. This is an additional mechanism by which platelet-leukocyte aggregates can also generate ROS. In our study, we were able to discriminate between platelet-originated ROS generation and leukocytemediated ROS production by measuring CD45+ versus CD45events This approach enabled us to demonstrate that leukocyte contamination of PCs is the main source of ROS of non-platelet origin. With this simple technique, we showed that the residual leukocytes in PCs can express much higher levels of DHR 123 (ROS generation) than single platelets, especially when they form aggregates with platelets. This observation points to the importance of leukoreduction for platelet products as it can significantly reduce ROS generation during the storage of platelet concentrates. Furthermore, given the fact that ROS also act as activating signaling molecules in cells, we compared the levels of DHR 123 expression in P-selectin+ or CD40L+cell populations versus those cells not expressing Pselectin or CD40L in order to unambiguously demonstrate direct correlation of platelet pro-inflammatory release and ROS generation. Intriguingly, we found high levels of DHR 123 expression in cells positively expressing these pro-inflammatory molecules and a non-detectable ROS generation in cells which were negative for Pselectin or CD40L. Our data also confirmed that agonist-like TRAP which significantly induces platelet granule release [10] also increases ROS generation in platelets. These observations not only confirm the association of ROS generation with platelet activation but also suggest a direct correlation between ROS production and platelet pro-inflammatory state during storage, which was also supported by the fact that the expression of P-selectin or DHR 123 is higher in platelet-leukocyte aggregates than in single platelets. This also shed light on the platelet-leukocyte crosstalk during storage and the possible role of residual leukocytes in the development of PSLs via ROS generation. To complete the story, further analysis of either soluble P-selectin or CD40L in stored platelets also con- firmed a direct correlation between the increasing levels of these pro-inflammatory shed molecules and the incrementing levels of DHR 123 expression in platelets. As it is already known from several studies [25] that the soluble form of CD40L increases intracellular ROS levels the correlation of ROS generation and soluble CD40L found in the present study is not surprising.

\section{Conclusion}

Our data confirmed increasing levels of intracellular ROS generation in both platelets and platelet-leukocyte aggregates during PC storage. ROS generation is directly correlated with platelet activation and granule release while platelets with no pro-inflammatory phenotype do not produce significant amounts of ROS. Considering the fact that ROS generation during platelet storage induces platelet activation and PSL, pharmacologic quenching of ROS production or scavenging of ROS molecules may protect banked platelets from PSL while promoting their in vivo functionality following transfusion. The inhibition of ROS generation may also reduce adverse transfusion reactions related to secreted inflammatory molecules.

\section{Acknowledgment}

This work was part of Dr. Ghasemzadeh's approved project (No.1393-0133-1781) supported by Iranian blood transfusion organization and High Institute for Research and Education in Transfusion Medicine in Iran.

\section{Disclosure Statement}

The authors declare no conflict of interests. 


\section{References}

1 Ohto H, Nollet KE: Overview on platelet preservation better controls over storage lesion. Transfus Apheresis Sci 2011;44:321-325.

2 Nassaji F, Ghasemzadeh M, Jamaat ZP, Hosseini E: The expression levels of platelet adhesive receptors in PRP derived platelet concentrates during storage. Tehran Univ Med J 2016;74:16-24.

3 Jamaat ZP, Hosseini E, Ghasemzadeh M: The expression loss of GPIba due to ectodomain shedding in PRP derived platelet concentrates during storage. Tehran Univ Med J 2016;74:92-98.

4 Cardigan R, Williamson L: The quality of platelets after storage for 7 days. Transfus Med 2003;13:173-187.

5 Leaver H, Schou A, Rizzo M, Prowse C: Calcium-sensitive mitochondrial membrane potential in human platelets and intrinsic signals of cell death. Platelets 2006;17:368-377.

6 Perrotta PL, Perrotta CL, Snyder EL: Apoptotic activity in stored human platelets, Transfusion 2003;43: 526535.

7 Skripchenko A, Kurtz J, Moroff G, Wagner SJ: Platelet products prepared by different methods of sedimentation undergo platelet activation differently during storage. Transfusion 2008;48:1469-1477.

8 Othman M, Labelle A, Mazzetti I, Elbatarny HS, Lillicrap D: Adenovirus-induced thrombocytopenia: the role of von Willebrand factor and P-selectin in mediating accelerated platelet clearance. Blood 2007;109: 2832-2839.

9 Leytin V, Allen DJ, Gwozdz A, Garvey B, Freedman J: Role of platelet surface glycoprotein Ibalpha and P-selectin in the clearance of transfused platelet concentrates. Transfusion 2004;44:1487-1495.

10 Hosseini E, Ghasemzadeh M, Nassaji F, Jamaat ZP GPVI modulation during platelet activation and storage: its expression levels and ectodomain shedding compared to markers of platelet storage lesion. Platelets 2016;28:498-508.

11 Sahler J, Spinelli S, Phipps R, Blumberg N: CD40 ligand (CD154) involvement in platelet transfusion reactions. Transfus Clin Biol 2012;19:98-103.

12 Refaai MA, Phipps RP, Spinelli SL, Blumberg N: Platelet transfusions: impact on hemostasis, thrombosis, inflammation and clinical outcomes. Thromb Res 2011;127:287-291.

13 Wachowicz B, Olas B, Zbikowska H, Buczynski A: Generation of reactive oxygen species in blood platelets. Platelets 2002;13:175-182.

14 Violi F, Pignatelli P: Platelet oxidative stress and thrombosis. Throm Res 2012;129:378-381.

15 Pignatelli P, Pulcinelli FM, Lenti L, Gazzaniga PP Violi F: Hydrogen peroxide is involved in collageninduced platelet activation. Blood 1998;91:484-490.

16 Sugamura K, Keaney JF: Reactive oxygen species in cardiovascular disease. Free Radic Biol Med 2011;51: 978-992.
17 Dayal S, Wilson KM, Motto DG, Miller FJ, Chauhan AK, Lentz SR: Hydrogen peroxide promotes aging-related platelet hyperactivation and thrombosis. Circulation 2013;127:1308-1316.

18 Pignatelli P, Carnevale R, Di Santo S, Bartimoccia S, Sanguigni V, Lenti L, Finocchi A, Mendolicchio L, Soresina AR, Plebani A: Inherited human gp91phox deficiency is associated with impaired isoprostane formation and platelet dysfunction. Arterioscler Thromb Vasc Biol 2011;31:423-434.

19 Pignatelli P, Carnevale R, Pastori D, Cangemi R, Napoleone L, Bartimoccia S, Nocella C, Basili S, Violi F: Immediate antioxidant and antiplatelet effect of atorvastatin via inhibition of Nox2. Circulation 2012; 126:92-103.

20 Krötz F, Sohn HY, Gloe T, Zahler S, Riexinger T, Schiele TM, Becker BF, Theisen K, Klauss V, Pohl U: NAD (P) $\mathrm{H}$ oxidase-dependent platelet superoxide anion release increases platelet recruitment. Blood 2002;100:917-924.

21 Krötz F, Sohn H-Y, Pohl U: Reactive oxygen species players in the platelet game. Arterioscler Thromb Vasc Biol 2014;24:1988-1996.

22 Noubade R, Wong K, Ota N, Rutz S, Eidenschenk C, Valdez PA, Ding J, Peng I, Sebrell A, Caplazi P, DeVoss J, Soriano RH, Sai T, Lu R, Modrusan Z, Hackney J, Ouyang W: NRROS negatively regulates reactive oxygen species during host defence and autoimmunity. Nature 2014;509:235-239.

23 Zhang K, Kaufman RJ: From endoplasmic-reticulum stress to the inflammatory response. Nature 2008;454: 455-462.

24 Zhou R, Yazdi AS, Menu P, Tschopp J: A role for mitochondria in NLRP3 inflammasome activation. Nature 2011;469:221-225.

25 Rizvi M, Pathak D, Freedman JE, Chakrabarti S: CD40-CD40 ligand interactions in oxidative stress, inflammation and vascular disease. Trends Mol Med 2008; 14:530-538.

26 Vanichakarn P, Blair P, Wu C, Freedman JE, Chakrabarti S: Neutrophil CD40 enhances platelet-mediated inflammation. Thromb Res 2008;122:346-358.

27 Kulkarni S, Woollard KJ, Thomas S, Oxley D, Jackson SP: Conversion of platelets from a proaggregatory to a proinflammatory adhesive phenotype: role of PAF in spatially regulating neutrophil adhesion and spreading. Blood 2007;110:1879-1886.

28 Ghasemzadeh M, Kaplan ZS, Alwis I, Schoenwaelder SM, Ashworth KJ, Westein E, Hosseini E, Salem HH, Slattery R, McColl SR: The CXCR1/2 ligand NAP-2 promotes directed intravascular leukocyte migration through platelet thrombi. Blood 2013;121:4555-4566.

29 Avendano A, Sales-Pardo I, Marin L, Marin P, Petriz J: Oxidative burst assessment and neutrophil-platelet complexes in unlysed whole blood. J Immunol Methods 2008;339:124-131.
30 Chakrabarti S, Vitseva O, Iyu D, Varghese S, Freedman JE: The effect of dipyridamole on vascular cell-derived reactive oxygen species. J Pharmacol Exp Ther 2005, 315:494-500.

31 Vitseva O, Varghese S, Chakrabarti S, Folts JD, Freedman JE: Grape seed and skin extracts inhibit platelet function and release of reactive oxygen intermediates. J Cardiovasc Pharmacol 2005;46:445-451.

32 Rothe G, Emmendorffer A, Oser A, Roesler J, Valet G: Flow cytometric measurement of the respiratory burst activity of phagocytes using dihydrorhodamine 123. J Immunol Methods 1991;138:133-135.

33 Yee J, Giannias B, Kapadia B, Chartrand L, Christou NV: Exudative neutrophils. Modulation of microbicidal function in the inflammatory microenvironment. Arch Surg 1994;129:99-105.

34 Khan SY, Kelher MR, Heal JM, Blumberg N, Boshkoy LK, Phipps R, Gettings KF, McLaughlin NJ, Silliman CC: Soluble CD40 ligand accumulates in stored blood components, primes neutrophils through CD40, and is a potential cofactor in the development of transfusionrelated acute lung injury. Blood 2006;108:2455-2462.

35 Bakdash N, Williams MS: Spatially distinct production of reactive oxygen species regulates platelet activation, Free Radic Biol Med 2008;45:158-166.

36 Violi F, Sanguigni V, Carnevale R, Plebani A, Rossi P Finocchi A, Pignata C, De Mattia D, Martire B, Pietrogrande MC, Martino S, Gambineri E, Soresina AR, Pignatelli P, Martino F, Basili S, Loffredo L: Hereditary deficiency of gp91(phox) is associated with enhanced arterial dilatation: results of a multicenter study. Circulation 2009;120:1616-1622.

37 Manasa K, Vani R Influence of oxidative stress on stored platelets. Adv Hematol 2016;2016:4091461.

38 Perales Villarroel JP, Figueredo R, Guan Y, Tomaiuolo M, Karamercan MA, Welsh J, Selak MA, Becker LB, Sims C: Increased platelet storage time is associated with mitochondrial dysfunction and impaired platelet function. J Surg Res 2013;184:422-429.

39 Skripchenko A, Myrup A, Thompson-Montgomery D Awatefe H, Wagner SJ: Mitochondrial dysfunction of platelets stored in first- and second-generation containers is, in part, associated with elevated carbon dioxide levels. Transfusion 2011;51:371-379.

40 Bonomini M, Stuard S, Carreno MP, Settefrati N, Santarelli P, Haeffner-Cavaillon N, Albertazzi A: Neutrophil reactive oxygen species production during hemodialysis: role of activated platelet adhesion to neutrophils through P-selectin. Nephron 1997;75:402-411.

41 Ghasemzadeh M, Hosseini E: Platelet-leukocyte crosstalk: linking proinflammatory responses to procoagulant state. Thromb Res 2013:131:191-197.

42 Ghasemzadeh M, Hosseini E: Intravascular leukocyte migration through platelet thrombi: directing leukocytes to sites of vascular injury. Thromb Haemost 2015;113:1224-1235. 